\title{
The Effect of Sulphoraphane on Brain Glucose Uptake during Neonatal Hypoxic-Ischemic Encephalopathy in Newborn Rats
}

Special Issue Article

\author{
${ }^{1}$ Institute of Experimental Pharmacology and \\ Toxicology, Centre of Experimental Medicine, Slovak \\ Academy of Sciences, Bratislava, Slovak Republic \\ Institute of Physiology, Czech Academy \\ of Sciences, Prague, Czech Republic
}

Kapoor, S. ${ }^{1,2 \varpi}$, Kala, D. ${ }^{2}$, Svoboda, J. ${ }^{2}$, Brnoliakova, Z. ', Otahal, J. ${ }^{2}$

Received 22 June, 2021, accepted 4 August, 2021

Abstract Neonatal hypoxic-ischemic insult (HII) is one of the leading causes of morbidity and mortality in newborns. It has long-term consequences and represents a socioeconomic burden. It is an urgent issue in current neonatology. The aim of the present pilot study was to evaluate the possible effect of sulforaphane on brain glucose uptake expressed as $18 \mathrm{~F}$-fluorodeoxyglucose (18F-FDG) activity at the acute, subacute, and subchronic time intervals after the experimental perinatal HII in rats. Significant protection has been observed in the hippocampus 5 weeks after the insult as represented by normalisations of interhemispheric ratio of measured 18F-FDG activity. In conclusion, positron emission tomography (PET) with 18F-FDG revealed a protective effect of SFN on glucose metabolism in the subchronic phase after HII. Further research within the field of neonatal HII in newborn rats will be necessary.

Keywords hypoxic-ischemic insult-18F-FDG (fluorodeoxyglucose) activity-oxidative stress-glucose metabolism-sulforaphane

\section{INTRODUCTION}

Neonatal hypoxic ischemic insult (HII) and consequent hypoxic ischemic encephalopathy (HIE) is a serious complication in newborns (Allen, 2012). Up to $15 \%$ to $20 \%$ of sufferers die in the early postnatal period. Infants who survive hypoxiaischemia, frequently develop neurological sequelae later in life, such as cerebral palsy, epilepsy, developmental delay, cognitive impairment, and behavioural disorders (Edwards et al., 2018). The pathologic events of HII are the result of impaired cerebral blood flow and deficient oxygen delivery to the brain, with resulting primary and secondary energy failure. Nonetheless, the pathophysiologic processes of $\mathrm{HI}$ are complex and evolve over time (Allen, 2012). Oxidative stress and inflammation are important pathophysiological factors which might impair various cellular targets, including the glucose metabolism pathway in both the acute and chronic periods after HII. The latest studies show that current treatment is only partially effective and infants still suffer from severe brain damage and neurologic impairments (Frajewicki et al., 2021). Nrf2 (nuclear factor erythroid 2-related factor 2) is a transcription factor that regulates a group of genes involved in antioxidative action and interacts with glucose metabolism (Heiss et al., 2013). Sulforaphane (SFN) is not a direct antioxidant, but rather regulates expression of endogenous antioxidative enzymes by the Nrf2 pathway (Kubo et al., 2017). It can protect the brain after onset of injury (Dang et al., 2012), and as well as during long-term recovery from cerebral ischemia (Liu et al., 2019). The aim of the present pilot study was to evaluate an effect of sulphoraphane on brain glucose uptake expressed as 18F-FDG activity in the experimental model of perinatal HII.

\section{MATERIAL AND METHODS}

In this pilot study, 6-day-old Sprague-Dawley rat pups, both male and female ( $\sim 18 \mathrm{~g}$ weight), were used $(\mathrm{N}=24$ : male, $n=12$; female, $n=12$ ). The protocol for the experiment was approved by the Animal Care and Use Committee in the Institute of Physiology, Czech Academy of Sciences. Animals were randomly divided into 4 groups: SHAM $(n=4)$, SHAM+SFN $(n=4)$, HIE $(n=8)$, and HIE + SFN ( $n=8)$. The number of animals 
within SHAM control groups $(n=4)$ was lower compared to those HIE ( $n=8)$ because of the elaborate and challenging experimentation pattern as well as the financial limitations of the pilot study. On day 6, sulforaphane i.p. ( $5 \mathrm{mg} / \mathrm{kg}$ b.w.) was administered in SHAM+SFN $(n=4)$, and HIE + SFN $(n=8)$. Perinatal HIl was induced on 7-day-old rat pups according to the Rice - Vannucci model (Vannucci and Vannucci, 2005) in $\mathrm{HIE}$ and $\mathrm{HIE}+\mathrm{SFN}$ groups. Briefly, in isoflurane-anaesthetised rat pups a left common carotid artery was isolated and ligated. After a short recovery animals were incubated in a normobaric hypoxic chamber $\left(\mathrm{pO}_{2} 8 \%\right)$ for 90 minutes. SHAM and SHAM+SFN groups underwent only the surgical procedure without hypoxia exposure and served as controls. Micro-CT and positron emission tomography ( $\mu \mathrm{CT} / \mathrm{PET}$ ) scans were performed to assess brain glucose uptake after $24 \mathrm{~h}, 1$ week, and 5 weeks of HIE with pretreatment with sulphoraphane in perinatal HII in particular groups of animals. The radioactive tracer 18F-FDG was used for the measurement of biodistribution and uptake of glucose in the brain. An activity of 10 to $20 \mathrm{MBq}$ of 18F-FDG (according to weight and age) in total volume 200 microliters (dissolved in sterile PBS) was injected into the jugular vein and after 45 minutes of bio-distribution, pups were anaesthetised and scanned in PET (30mins) a microCT. The data were analysed in PMOD Technologies LLC, Switzerland software with the use of Schiffer's magnetic resonance imaging (MRI) rat brain atlas to assess $18 \mathrm{~F}-\mathrm{FDG}$ activity in the individual brain regions (Sánchez et al., 2013). All data were statistically analysed using one-way ANOVA in Sigmaplot software and data were expressed as mean \pm SEM. Statistical significance was indicated at $\mathrm{P}$ less than 0.05 .

\section{RESULTS}

Alterations in FDG (fluorodeoxyglucose) uptake were evaluated after $24 \mathrm{hr}, 1$ week, and 5 weeks of perinatal HII. 18F-FDG activity was measured and calculated as leftto-right ratio (L/R) in both hemispheres for an individual neuroanatomical structure as per MRI atlas. The significant changes were observed in the L/R ratio in the group of SHAM+SFN after $24 \mathrm{hr}$ and after 1 week of HIl, while it was decreased 5 weeks after HII in comparison to the SHAM group. After $24 \mathrm{hr}$, significant differences were revealed in the contralateral ratio 18F-FDG activity in HIE+SFN and HIE group compared to SHAM+SFN group; see Fig. 1A. After 1 week of HIE, there was significant difference in the SHAM, HIE, $\mathrm{HIE}+\mathrm{SFN}$ groups compared to SHAM+SFN group; see Fig. 1B. Finally, after 5 weeks, there was significant difference also in SHAM+SFN and HIE group compared to the SHAM group. Moreover, we found the significant difference also in HIE+SFN compared to theHIE group; see Fig. 1C.

\section{DISCUSSION}

Our data indicate that SFN has an important impact in the acute phase after the HII in newborns. 18F-FDG PET imaging
A)

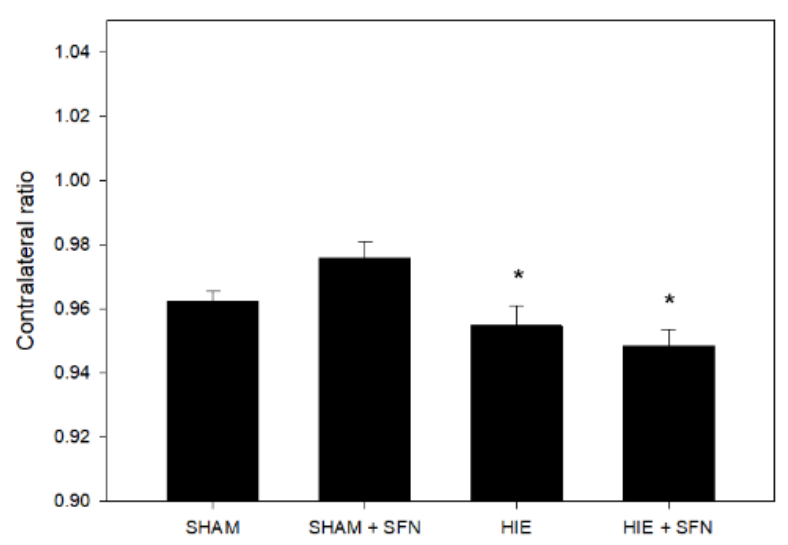

B)

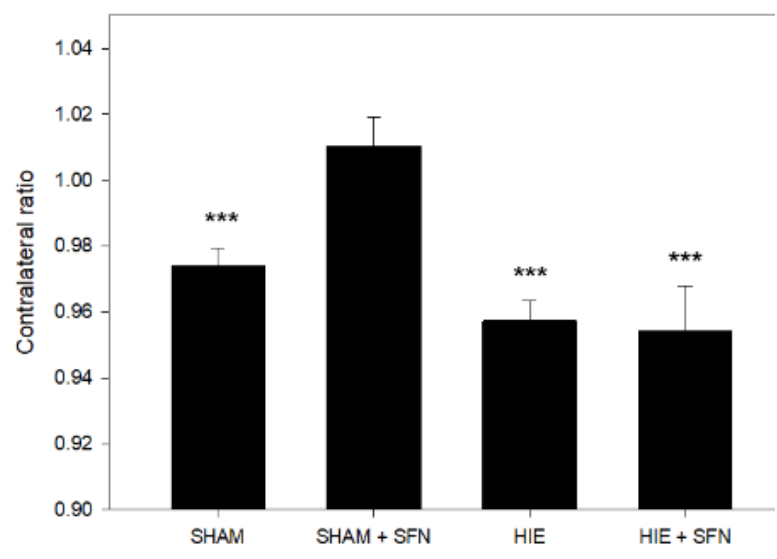

C)

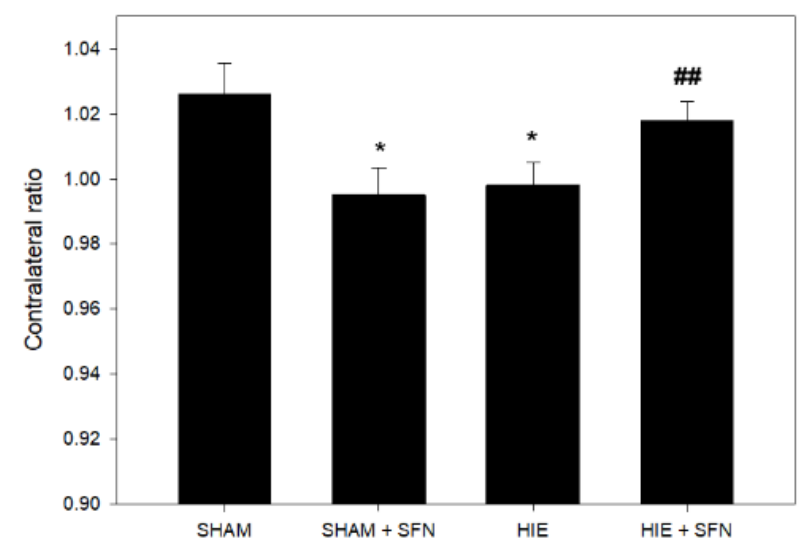

Figure 1: Contralateral ratio of 18F-FDG activity after $24 \mathrm{hr}$ (A), 1 week (B), 5 weeks (C) after HIE. ANOVA statistics: data were expressed as mean \pm SEM. $(A){ }^{*} P<0.05$ HIE $+S F N$ and HIE vs SHAM+SFN; (B) ${ }^{* * *} P<0.001$ HIE+SFN and HIE and SHAM vs SHAM+SFN; (C) *P<0.05 SHAM+SFN and HIE vs SHAM; \# \# $P<0.01$ HIE + SFN vs HIE. 
evaluates the alterations of cerebral glucose uptake activity after HII. The most typical affected regions in the hypoxic ischemic model are the hippocampus and surrounding cortex (Huang and Castillo, 2008). The mechanism of the SFN action has to be elucidated by further studies; however, previously published data suggest several possible pathways. The study of Danilov et al. (2009) revealed that sulforaphane protects astrocytes from delayed death caused by oxygen and glucose deprivation in an ischemia/refusion paradigm by increasing mRNA, protein levels, and increasing Nrf2 and HO-1 (heme oxygenase) expression. SFN activates the Nrf2/ ARE (antioxidant response element) pathway, which reduces infarct volume, oxidative stress, and apoptotic cell numbers. It has also been demonstrated to have a prominent impact on motor performance in behavioral deficits (Giacoppo et al., 2015). SFN exhibits neuroprotective properties in animal models of neurodegeneration as it crosses the blood-brain barrier and accumulates in the central nervous system (Tarozzi et al., 2013). Enhanced production of Nrf2-driven genes preserves the blood-brain barrier after brain injury (Guerrero-Beltrán et al., 2012). Narayanaswami et al. (2018) declared that PET imaging plays a key role in central nervous system discoveries concerning visualising and measuring changes within metabolic processes. Since 18 F-FDG is transported into metabolically active tissue via the same transport pathways as glucose, active regions express higher signal, which allows one to distinguish highly active regions (such as inflammation or tumour) from those affected by necrosis (Svoboda et al., 2019). Additionally, recent preclinical studies suggest that 18F-FDG PET can identify affected regions in neurodegenerative disorders (Narayanaswami et al., 2018).

\section{CONCLUSION}

In our pilot study, we demonstrated that pretreatment with SFN led to significant protection in the hippocampus 5 weeks after the insult as represented by both normalisations of interhemispheric ratio and increase in measured 18F-FDG activity. Although sulphoraphane is acting mainly through the Nrf2 pathway, further research will be necessary to elucidate the exact mechanism of action in HII in newborn rats.

\section{ACKNOWLEDGEMENTS}

This pilot study was supported by grants: EU project ITMS2014+313021Y920, APVV-18-0336, VEGA 2/0104/21, Czech Science Foundation 18-07908 and the IPHYS facility within the MEYS CR (Large RI Project LM2018129 CzechBiolmaging).

\section{References}

[1] Allen KA. Hypoxic Ischemic Encephalopathy: Pathophysiology Experimental Treatments. 2012;11(3):125-33.

[2] Edwards AB, Anderton RS, Knuckey NW, Meloni BP. Perinatal hypoxic-ischemic encephalopathy and neuroprotective peptide therapies: A case for cationic arginine-rich peptides (CARPs). Brain Sci. 2018;8(8):15-20.

[3] Frajewicki A, Laštůvka Z, Borbélyová V, Khan S, Jandová K, Janišová $K$, et al. Perinatal hypoxic-ischemic damage: review of the current treatment possibilities. Physiol Res. 2021;69:S379_ 401.

[4] Heiss EH, Schachner D, Zimmermann K, Dirsch VM. Glucose availability is a decisive factor for Nrf2-mediated gene expression. Redox Biol. 2013;1(1):359-65.

[5] Kubo E, Chhunchha B, Singh P, Sasaki H, Singh DP. Sulforaphane reactivates cellular antioxidant defense by inducing Nrf2/ ARE/Prdx6 activity during aging and oxidative stress. Sci Rep. 2017;7(1):1-17.

[6] Dang J, Brandenburg LO, Rosen C, Fragoulis A, Kipp M, Pufe T, et al. Nrf2 expression by neurons, astroglia, and microglia in the cerebral cortical penumbra of ischemic rats. J Mol Neurosci. 2012;46(3):578-84.

[7] Liu L, Locascio LM, Doré S. Critical Role of Nrf2 in Experimental Ischemic Stroke. Front Pharmacol. 2019; 10:153

[8] Vannucci RC, Vannucci SJ. Perinatal hypoxic-ischemic brain damage: Evolution of an animal model. Developmental Neuroscience. 2005.
[9] Sánchez F, Orero A, Soriano A. ALBIRA: A small animal PET / SPECT / CT imaging system. 2013;40(5):1-11.

[10] Huang BY, Castillo M. Hypoxic-Ischemic brain injury: Imaging findings from birth to adulthood. Radiographics. 2008;28(2):417-39.

[11] Danilov CA, Chandrasekaran K, Racz J, Soane L, Zielke C, Fiskum G. Sulforaphane protects astrocytes against oxidative stress and delayed death caused by oxygen and glucose deprivation. Glia. 2009; 57(6): 645-656.

[12] Giacoppo S, Galuppo M, Montaut S, lori R, Rollin P, Bramanti P, et al. An overview on neuroprotective effects of isothiocyanates for the treatment of neurodegenerative diseases. Fitoterapia. 2015;106:12-21.

[13] Tarozzi A, Angeloni C, Malaguti M, Morroni F, Hrelia S, Hrelia P. Sulforaphane as a potential protective phytochemical against neurodegenerative diseases. Oxid Med Cell Longev. 2013;2013: 415078.

[14] Guerrero-Beltrán CE, Calderón-Oliver M, Pedraza-Chaverri J, Chirino YI. Protective effect of sulforaphane against oxidative stress: Recent advances. Exp Toxicol Pathol. 2012;64(5):503-8.

[15] Narayanaswami V, Dahl K, Bernard-Gauthier V, Josephson L, Cumming $P$, Vasdev N. Emerging PET radiotracers and targets for imaging of neuroinflammation in neurodegenerative diseases: Outlook Beyond TSPO. Mol Imaging. 2018;17:1-25.

[16] Svoboda J, Litvinec A, Kala D, Pošusta A, Vávrová L, Jiruška $P$, et al. Strain differences in intraluminal thread model of middle cerebral artery occlusion in rats. Physiol Res. 2019;68(1):37-48. 